\title{
BMJ Open Feasibility of a 6-month pilot randomised controlled trial of resistance training on cognition and brain health in Canadian older adults at-risk for diabetes: study protocol
}

\author{
Joyla A Furlano (D) , ${ }^{1}$ Lindsay S Nagamatsu ${ }^{2}$
}

To cite: Furlano JA, Nagamatsu LS. Feasibility of a 6-month pilot randomised controlled trial of resistance training on cognition and brain health in Canadian older adults at-risk for diabetes: study protocol. BMJ Open 2019;9:e032047. doi:10.1136/ bmjopen-2019-032047

- Prepublication history for this paper is available online. To view these files, please visit the journal online (http://dx.doi org/10.1136/bmjopen-2019032047).

Received 04 June 2019 Revised 18 August 2019 Accepted 10 September 2019

Check for updates

(c) Author(s) (or their employer(s)) 2019. Re-use permitted under CC BY-NC. No commercial re-use. See rights and permissions. Published by BMJ.

${ }^{1}$ Neuroscience, Western University, London, Ontario, Canada

${ }^{2}$ Kinesiology, Western University, London, Ontario, Canada

Correspondence to Dr Lindsay S Nagamatsu; lindsay.nagamatsu@uwo.ca

\section{ABSTRACT}

Introduction Type 2 diabetes (T2D) is associated with cognitive deficits and increased risk of dementia, and thus individuals at high risk for T2D (ie, those who are overweight or prediabetic) are also at greater risk for cognitive decline. Aerobic exercise is known to preserve and improve cognitive function, but the effects of resistance training (RT) are much less known in older adults. Moreover, research on the effects of RT on cognition and brain health (structure and function) in older adults at-risk for diabetes is limited. To address this question, a 6-month RT intervention is needed. Importantly, before conducting a full-scale randomised controlled trial (RCT), we are conducting a feasibility pilot study to assess potential recruitment rates, adherence and retention in this specific population.

Methods and analysis We are conducting a 6-month, thrice-weekly RT RCT. Participants (aged 60-80; sedentary; fasting plasma glucose of $6.1-7.0 \mathrm{mmol} / \mathrm{L}$ or body mass index $\geq 25$ ) are randomised into one of two groups: (1) RT or (2) balance and tone (control). Based on other exercise trials using a similar population, we will consider our trial feasible if we have adherence and retention at $70 \%$. Recruitment rate will be measured as time it takes to enrol 20 participants. To assess behavioural and MRI data, we will report descriptive statistics and estimation using a $95 \% \mathrm{Cl}$.

Ethics and dissemination Our study has received ethics approval from the Health Sciences Research Ethics Board at Western University. As this is a small pilot study, data will only be made available to other researchers on request. Results from this study will be disseminated via academic publication.

Trial registration number NCT03254381

\section{INTRODUCTION}

Dementia affects approximately 47 million people worldwide ${ }^{1}$ and is a major cause of disability among older adults. ${ }^{2}$ One of the largest modifiable risk factors for dementia is type 2 diabetes (T2D), ${ }^{3}$ a long-term metabolic disorder characterised by high blood sugar. Research has shown that older adults
Strengths and limitations of this study

- This is the first study to assess the feasibility of conducting a randomised controlled trial of resistance training to improve cognitive function in older adults at-risk for diabetes.

- The results from this feasibility study will inform our large-scale study of effective strategies to work with this at-risk population.

- Participants are not blinded to their treatment group. Thus, maintaining retention for the control group may be especially challenging, as participants in similar studies are often more interested in being in the active exercise group.

- Since our study is not adequately powered, statistical analysis is limited; we will be reporting descriptive statistics.

with T2D experience cognitive decline and neural atrophy beyond normative ageing, and therefore are at high risk for developing dementia. $^{45}$

Importantly, older adults at-risk for developing T2D (ie, overweight or prediabetic individuals) already show some evidence of cognitive decline. Marseglia and colleagues found that compared with healthy controls, older adults with prediabetes perform significantly worse on cognitive tasks assessing memory, perceptual speed and category fluency. ${ }^{5}$ Similarly, cross-sectional studies have shown that overweight older adults (body mass index $(\mathrm{BMI}) \geq 25)$ experience deficits in memory and global cognition compared with older adults with BMI $<25 .{ }^{67}$ Thus, older adults at-risk for T2D experience cognitive deficits that may worsen as the progression to T2D occurs, and intervention strategies aimed at improving cognitive function in this at-risk group may represent an ideal time point to prevent or delay future decline. 
One promising lifestyle intervention that may improve neurocognitive function is exercise. Previous research has found that exercise can improve performance in several cognitive domains, including memory and executive function ${ }^{8-10}$ and can lead to changes in brain function ${ }^{11}$ and structure. ${ }^{12} 13$ Of particular relevance to our study, Baker and colleagues found that aerobic training (AT), defined as exercise with the goal of increasing cardiovascular capacity, improves cognition in older adults at-risk for diabetes. ${ }^{14}$ In this study, older adults with glucose intolerance showed improvements in memory and executive function after a 6-month aerobic exercise intervention four times per week. However, this study did not assess functional or structural brain changes that may be associated with these effects.

While the majority of research on exercise and cognition has focused on AT, there are other forms of exercise that may also be beneficial for older adults. For example, resistance training (RT), defined as exercise with the goal of increasing muscle mass, has been shown to improve cognitive function and brain health. In one study, sedentary women with mild cognitive impairment improved their executive function, associative memory and functional activation in key brain regions following 6 months of progressive RT. ${ }^{15}$ Furthermore, RT has additional benefits for older adults, such as reducing sarcopenia, ${ }^{16}{ }^{17}$ and is useful for older adults who may suffer from physical and/or mobility impairments and cannot participant in AT, thus providing older adults with an alternative form of exercise that may benefit cognition and brain health.

Animal research on RT has found that this type of exercise may increase insulin-like growth factor 1 (IGF-1), ${ }^{18} 19$ a growth factor known to have neuroprotective abilities associated with improved cognitive function. ${ }^{20}$ Studies have shown that low levels of IGF-1 are associated with impaired glucose tolerance and a higher risk of T2D. For example, Friedrich and colleagues found that low levels of IGF-1 serum concentrations are associated with insulin resistance in adults of various ages. ${ }^{21}$ Schneider $e t$ al measured IGF-1 levels in non-diabetic individuals and assessed incident diabetes during follow-up, and found that patients with low IGF-1 levels were at an increased risk of glucose intolerance and developing diabetes. ${ }^{22}$ Based on this combined knowledge, RT may be particularly useful in not only improving insulin resistance and reducing one's risk of developing T2D, but also increasing cognitive function in this population.

To our knowledge, research has not yet examined the effects of RT on cognition in older adults at-risk for diabetes. To address whether RT may benefit cognitive function and brain health in older adults at-risk for diabetes, a randomised controlled trial (RCT) is needed. However, there are potential barriers towards conducting such an intervention in this population-namely, the ability to recruit sedentary older adults at-risk for diabetes and adherence and retention rates over a 6 -month period. Therefore, before proceeding with a full-scale RCT, we are conducting a feasibility pilot study. The primary aim of our current study is to determine the feasibility of conducting a 6-month, thrice-weekly exercise intervention in older adults at-risk for diabetes.

\section{METHODS}

\section{Study design}

We are conducting a single-blinded pilot RCT to examine the feasibility of a 6-month, thrice-weekly exercise intervention. Assessments will be made at baseline, midpoint (3 months) and trial completion (6 months). Cognitive and physical assessments will be conducted on campus at Western University, London, Ontario, along with all exercise sessions. Magnetic resonance imaging (MRI) will be done using a 3T Siemens scanner at Robarts Research Institute. Participant enrolment is ongoing. The Standard Protocol Items: Recommendations for Interventional Trials and Consolidated Standards of Reporting Trials checklists have been completed.

\section{Patient and public involvement}

The public was not directly involved in the development of the research question, outcome measures or design of this study. The burden of the intervention will be assessed by asking participants to complete an 'end of study questionnaire' where they have the opportunity to report their subjective experiences during the intervention.

\section{Participants}

We will recruit participants from the city of London, Ontario (urban community) via flyers, newspaper/ online advertisements and word-of-mouth, as well as by giving short talks at community centres. We have chosen these recruitment methods based on the success of other studies at Western University in recruiting older adults. We will have ongoing enrolment in the study until we reach our target sample size. Our target sample size is 20 participants (10 per arm), which is based on the aims of this pilot study - to gauge the rate of recruitment and adherence and retention levels, as well as determine any unanticipated issues that would need to be resolved for a full-scale RCT. This is also the recommended sample size for a pilot study where the full-scale trial would have an anticipated medium effect size with a power of $0.80 .^{23}$

To be included in our study, individuals must: (1) be community-dwelling, (2) be aged 60-80 years, (3) be 'at-risk' for diabetes, where they have one of the following: $\mathrm{BMI} \geq 25$ or blood glucose level of $6.1-7 \mathrm{mmol} / \mathrm{L}$, (4) score $>24 / 30$ on Mini-Mental State Examination (MMSE), (5) score $>6 / 8$ on the Lawton Activities of Daily Living scale, (6) have visual acuity of at least $20 / 40$, with or without corrective lenses, (7) speak and understand English fluently and (8) complete the Physical Activity Readiness Questionnaire and obtain physician's clearance to start a supervised exercise programme. We will exclude those who: (1) have a current medical condition for which exercise is contraindicated, (2) have participated regularly (>1/week) in resistance or AT in the last 6 months, 
(3) have been diagnosed with neurodegenerative disease (including dementia, Alzheimer's disease or Parkinson's disease), (4) have experienced a vascular incident (eg, stroke, myocardial infarction), (5) have been diagnosed with a psychiatric condition, (6) have untreated depression, (7) are currently on hormone replacement therapy, (8) have clinically significant peripheral neuropathy or severe musculoskeletal or joint disease, (9) are currently taking psychotropic medications or (10) are unable to participate in MRI (eg, metal or electronic implants, claustrophobic).

\section{Descriptive measures}

To describe our sample and assess eligibility, we will collect the following measures: (1) demographic information via questionnaires (age, sex, education, socioeconomic status), (2) fasting blood glucose using a glucometer; participants will be asked to fast 8 hours prior to testing, (3) height and weight to calculate BMI, (4) waist and hip circumference, (5) muscle strength (ie, assessing major muscle groups such as quadriceps, latissimus dorsi, hamstrings, pectoralis major, biceps, triceps, deltoids and trapezius muscles) using one repetition maximum assessment (described below) on the following machines: leg press, lat pull, leg curl, chest press, bicep curl, tricep curl and seated row, (6) sleep quantity and quality using a monthly questionnaire, (7) general levels of physical activity using the Physical Activity Scale for the Elderly, ${ }^{24}(8)$ balance and mobility using the Short Physical Performance Battery ${ }^{25}$ and the Timed Up and Go Test, ${ }^{26}$ (9) number and type of comorbidities using the Functional Comorbidity Index, ${ }^{27}$ (10) depression screening using the Geriatric Depression Scale, ${ }^{28}$ (11) aerobic fitness using the 6 min walk test ${ }^{29}$ and (12) global cognitive impairment screening using the $\mathrm{MMSE}^{30}$ and the Montreal Cognitive Assessment. ${ }^{31}$

\section{Primary outcome measures}

To assess feasibility of our intervention, our primary outcome measures are: (1) recruitment rate: time it takes to enrol 20 participants, (2) adherence: percentage of exercise classes attended over 6 months [ (number of classes attended/72 classes maximum) x 100] and (3) retention: percentage of participants enrolled in the study who complete the 6 month intervention. Attendance to exercise classes will be tracked by fitness instructors throughout the intervention. Based on other exercise trails using similar populations of older adults, we will consider our trial feasible if adherence and retention are at $70 \%{ }^{32} 33$ If participants miss sessions, we will follow-up with them via phone. On trial completion, we will not follow-up with participants as this is a pilot study.

\section{Secondary outcome measures}

Qualitative feasibility assessment

To complement our primary outcome measures, we will administer separate questionnaires to both participants and exercise trainers via email for their convenience.
Both questionnaires will gauge enjoyment and satisfaction with the study and will help to assess which aspect(s) of the exercise intervention went well and how we can improve the study in the future. Specific topics of the participant questionnaire include level of enjoyment and socialisation, while topics of the exercise trainer questionnaire include challenges faced in facilitating and quality of training. Additionally, both questionnaires assess reasons for joining the study and general feedback. The questionnaires feature 7-point Likert scale questions and open-ended questions, such as 'What was the most difficult part of the study?' (participant questionnaire) and 'In your opinion, is there anything that you would change to improve the study?' (exercise trainer questionnaire). These questionnaires will provide further information regarding feasibility of our trial as well as identify potential barriers and strategies for working with this population that can be implemented in the full-scale RCT.

\section{Cognitive function}

The purpose of the full-scale RCT will be to examine the effects of RT versus balance-and-tone (BAT; control group) on cognition and brain health (structure and function). Cognition will be assessed using the Alzheimer's disease Assessment Scale $11^{34}$ : a test made up of 11 tasks measuring various cognitive functions (eg, memory, language, praxis, attention) and used widely in clinical trials to assess cognitive dysfunction and Alzheimer's disease.

Related yet distinct components of executive function will be assessed using the following measures: (1) Trail Making Test Parts A and B-used to assess set-shifting, ${ }^{35}$ (2) Stroop Test-used to assess selective attention and conflict resolution $^{36}$ and (3) Digit Span Forward and Backward-used to assess working memory. ${ }^{37}$ In addition, memory and learning will be assessed using the Rey Auditory Verbal Learning Test, used to assess short-term auditory-verbal memory. ${ }^{38}$

\section{Magnetic resonance imaging}

To assess brain structure and function, participants will undergo an MRI scan in the 3T Siemens scanner at the Centre for Functional and Metabolic Mapping at Robarts Research Institute. The structural imaging protocol will include the following sequences: (1) diffusion tensor imaging to assess structure connectivity, (2) T2-weighted-Fluid-Attenuated Inversion Recovery (FLAIR) to assess pathology/disease and (3) TI-weighted imaging to assess regional volumes. T1-weighted images $(\mathrm{TR}=2300$, $\mathrm{TE}=2.98$, voxel size $=1 \times 1 \times 1$ ) will be used to calculate absolute and normalised brain volumes using Structural Image Evaluation using Normalisation of Atrophy-Cross-sectional (SIENAX) within FMRIB's Software Library (FSL V.5.0.10). In addition, left and right hippocampal volumes will be calculated via FIRST within FSL.

Functional imaging (fMRI) will be used to assess changes in functional activation over the 6-month intervention. Participants will complete an associative memory 
task during blood oxygen level dependent imaging (TR (repetition time) $=1000$, TE (echo time)-30, slice thickness $=2.5 \mathrm{~mm}$ ). Mean percentage signal change will be acquired from memory-related regions of interest, including the right and left hippocampi, posterior and anterior parahippocampal gyri, and the left and right medial temporal gyri. We will be assessing associative memory, the ability to remember the relationship between two items, as this is known to decline early in ageing and is a hallmark of early stages of dementia. ${ }^{39}$

The associative memory task ${ }^{10} 11$ is a mixed block/ event-related design. This task requires participants to view a series of photos, in which they encode and recall items separately (faces or places) and items in conjunction (faces matched with places). While performing the task inside the scanner, participants will use a two-button response pad. The task features six conditions: face encoding, face recognition, place encoding, place recognition, face-place encoding and face-place recognition. Participants will complete three blocks, each comprised of the six conditions with the order of conditions counterbalanced. Each condition features 12 separate photos; each of the 12 images appear for 3000 milliseconds, separated by a fixation cross appearing for $6000 \mathrm{~ms}$. During the encoding conditions, participants will be asked to indicate one of the following: (1) if the face shown is male or female, (2) if the background scenery contains liquid water or (3) if the face 'fits well' with the scenery (there is no correct answer to this question-this is used to assist participants in encoding the items in conjunction). Following each encoding condition, participants, in the recognition condition, will be asked to indicate whether they have previously seen each of the images during the encoding sequence. Each recognition block will contain six images from the encoding condition and six distractor images. Participants will complete practice trials of the memory task prior to completing the task in the scanner.

To assess functional networks, participants will also undergo a resting-state fMRI scan. During this 12-min scan, participants will be required to lay still in the scanner with their eyes open while starring at a fixation cross.

\section{Randomisation and blinding}

After baseline assessments, participants will be randomised (randomisation.com; random block sizes of 2, 4 and 6) into either the RT group or the BAT control group. The group allocation sequence will be held independently by the Primary Investigator. All assessments will be completed by blinded assessors. Participants will not be blinded to group assignment, but they will not have direct contact with the other exercise groups or be made aware of our hypotheses.

\section{Intervention}

Participants in both intervention arms will complete 60 min of exercise three times per week for 26 weeks. Exercise protocols have been adapted from those developed by Dr Teresa Liu-Ambrose and used successfully in similar interventions. ${ }^{101115}$ All exercise sessions will be led by trained fitness leaders from the School of Kinesiology at Western University.

\section{Resistance training}

Participants in this group will use seven programmable pressurised air weight machines (HUR equipment) along with free weights to target primary muscle groups (eg, quads, biceps, triceps and so on). In addition, they will complete mini-squats, mini-lunges and lunge walks. The first 3 weeks will be used to introduce participants to the machines and allow them to become familiar with their set-up and ensure proper form. One repetition maximum, a physical measure of an individual's maximum muscle strength during a single repetition, will be tested on all machines at the end of week three to determine the desired load for RT. To do this, participants will complete two sets of 6-8 reps of each exercise during each class, and training stimulus will be increased using the 7RM method-when two sets of 6-8 reps are completed with proper form and without discomfort. ${ }^{1011}$ The number of sets completed and the load lifted for each exercise will be recorded at every class for each participant.

\section{Control}

Participants in this group will engage in exercises which include stretching, range of motion exercises, basic corestrength exercises, balance exercises and relaxation techniques; only bodyweight will be applied (ie, no additional loading). These exercises have not been shown to significantly improve cognitive function. ${ }^{40}$ The BAT group serves to control for confounding variables such as socialisation, commitment to the programme and lifestyle changes associated with the exercise programme.

For both groups, activities outside of the intervention itself will not be restricted, but we will monitor physical activity levels throughout the 6 months through self-reported measures to account for any potential confounds that may influence results.

\section{Data analysis}

As this is a feasibility study, our primary aim is to assess whether a full-scale RCT would be feasible to conduct given recruitment, adherence and retention. Therefore, we will report the rate of recruitment and adherence/ retention throughout the trial. We will also include a qualitative analysis of the feasibility of our study, including barriers and strategies for working with this population. To do so, we will conduct a content analysis of our two questionnaires, in which we will categorise the data into concepts and identify patterns of responses.

Behavioural (cognitive assessments and physical measures) and MRI data will be assessed between groups over time. As this is a pilot study and thus is not adequately powered, we will be reporting descriptive statistics and estimation, using a $95 \% \mathrm{CI}$, to infer the magnitude of treatment effect for future power calculations to inform a full-scale intervention. MRI and fMRI data will be 
analysed in FSL to examine changes in brain structure (eg, hippocampal volume) and functional activation and network activity.

\section{ETHICS AND DISSEMINATION Monitoring}

While our study is low risk, adverse events associated with exercise or physical testing may occur, such as pain, discomfort, shortness of breath or injury. All participants will be monitored closely by instructors for symptoms of these occurrences during exercise sessions. Any adverse effects will be recorded and reported immediately. In the event of an injury, we will seek medical care immediately.

To assess fidelity of the exercise sessions, we will administer a questionnaire once per month in which a non-study affiliate will attend a randomly selected exercise class and assess both the RT and BAT sessions. Specifically, he/ she will follow a checklist assessing and reporting on the structure of the class, including the delivery of the exercises and the performance of the participants.

\section{Privacy}

We will collect the following personal identifiers: name, date of birth, telephone number and email. Since this is a 6-month RCT, we need to link participant baseline data with trial completion data. We will also need to keep in regular contact with participants throughout the study. Participants are free to withdraw from the intervention at any time.

All participants will be assigned a Participant ID number; identifiable information will be password-protected and kept on a password-protected computer. All study data will also be stored on a password-protected computer and backed up to an encrypted external hard drive (FireVault) that will be stored in the lab and only accessed by research personnel.

\section{Dissemination}

As this is a small pilot study, we will not be depositing our data in publicly available repositories. However, data will be made available to other researchers on request. Results from this study will be disseminated via academic publication and will be made available to participants directly on request.

\section{DISCUSSION}

We have presented the rationale and design of a pilot study assessing feasibility of an exercise RCT in older adults at-risk for diabetes. If shown to be feasible, a largescale RCT will be conducted, in which we aim to demonstrate whether 6 months of resistance exercise training will improve cognitive and brain health in this population. In our large-scale study, we will conduct a full process evaluation in order to assess whether the intervention is implemented as intended. If shown efficacious, lifestyle interventions, such as exercise, will provide low-cost and low-risk solutions to combat some of our largest health challenges, such as diabetes and associated diseases (dementia).

Through this pilot study, we hope to identify potential strategies and barriers towards working with this at-risk population that may benefit future intervention trials. We anticipate that recruitment will be difficult for several reasons. First, we have an extensive list of inclusion and exclusion criteria in order to reduce the variance within each group; however, this will impact the number of individuals who are able to participate in our study. To ensure we have enough participants in our study, we will include participants who are overweight and/or prediabetic (based on blood glucose level). In larger future trials, however, it would be ideal to separate these groups further to examine whether exercise differentially impacts cognition in these distinct groups of individuals.

Second, sedentary and overweight older adults might feel reluctant volunteering for a 6-month exercise programme. Specifically, going from not regularly exercising to committing to exercise three times per week for 6 months may seem daunting for some individuals. We will ensure potential participants are aware that exercise classes will start out at beginner level and will be supervised by trained leaders. We will also allow participants to bring a friend or spouse to the exercise classes to increase adherence and motivation. Our end-of-study questionnaires in this pilot phase will allow us to identify other barriers and potential solutions that we will be able to incorporate into the full-scale RCT.

\section{CONCLUSION}

We present the protocol for a pilot RCT aimed to assess the feasibility of a full-scale RCT examining the effects of exercise on cognition and brain health in older adults at-risk for diabetes. Exercise is a promising lifestyle intervention that may positively impact those most at-risk for cognitive decline, providing a scalable and cost-effective strategy to offer our ageing population.

Acknowledgements The authors thank the participants for their dedication and involvement in this study.

Contributors JAF and LSN devised the study. This manuscript was prepared and written by JAF, with edits and contributions from LSN. All authors read and approved the final manuscript.

Funding This study is funded through Western Strategies Support for CIHR Success.

Competing interests None declared.

Patient consent for publication Not required.

Ethics approval Our study has received ethics approval from the Health Sciences Research Ethics Board at Western University.

Provenance and peer review Not commissioned; externally peer reviewed.

Open access This is an open access article distributed in accordance with the Creative Commons Attribution Non Commercial (CC BY-NC 4.0) license, which permits others to distribute, remix, adapt, build upon this work non-commercially, and license their derivative works on different terms, provided the original work is properly cited, appropriate credit is given, any changes made indicated, and the use is non-commercial. See: http://creativecommons.org/licenses/by-nc/4.0/. 
ORCID iD

Joyla A Furlano http://orcid.org/0000-0003-4098-8351

\section{REFERENCES}

1 World Health Organization. 10 facts on dementia. Available: https:// www.who.int/features/factfiles/dementia/en/ [Accessed 15 Oct 2018].

2 National Institute on Aging. Dementia. Available: https://www.nia.nih. gov/health/topics/dementia [Accessed 9 Oct 2018].

3 Awad N, Gagnon M, Messier C. The relationship between impaired glucose tolerance, type 2 diabetes, and cognitive function. J Clin Exp Neuropsychol 2004;26:1044-80.

4 Cholerton B, Baker LD, Montine TJ, et al. Type 2 diabetes, cognition, and dementia in older adults: toward a precision health approach. Diabetes Spectr 2016;29:210-9.

5 Marseglia A, Fratiglioni L, Laukka EJ, et al. Early cognitive deficits in type 2 diabetes: a population-based study. J Alzheimers Dis 2016;53:1069-78.

6 Benito-León J, Mitchell AJ, Hernández-Gallego J, et al. Obesity and impaired cognitive functioning in the elderly: a population-based cross-sectional study (NEDICES). Eur J Neurol 2013;20:899-e77.

7 Gunstad J, Lhotsky A, Wendell CR, et al. Longitudinal examination of obesity and cognitive function: results from the Baltimore longitudinal study of aging. Neuroepidemiology 2010;34:222-9.

8 Zhu N, Jacobs Jr DR, Schreiner PJ, et al. Cardiovascular fitness and cognitive function in middle age. Neurology 2014;82:1339-46.

9 Heisz JJ, Clark IB, Bonin K, et al. The effects of physical exercise and cognitive training on memory and neurotrophic factors. $J$ Cogn Neurosci 2017;29:1895-907.

10 Liu-Ambrose T, Nagamatsu LS, Graf P, et al. Resistance training and executive functions: a 12-month randomized controlled trial. JAMA Intern Med 2010;170:170-8.

11 Liu-Ambrose T, Nagamatsu LS, Voss MW, et al. Resistance training and functional plasticity of the aging brain: a 12-month randomized controlled trial. Neurobiol Aging 2012;33:1690-8.

12 Erickson KI, Prakash RS, Voss MW, et al. Aerobic fitness is associated with hippocampal volume in elderly humans. Hippocampus 2009;19:1030-9.

13 Erickson KI, Voss MW, Prakash RS, et al. Exercise training increases size of hippocampus and improves memory. Proc Natl Acad Sci U S A 2011;108:3017-22.

14 Baker LD, Frank LL, Foster-Schubert K, et al. Aerobic exercise improves cognition for older adults with glucose intolerance, a risk factor for Alzheimer's disease. J Alzheimers Dis 2010;22:569-79.

15 Nagamatsu LS, Handy TC, Hsu L. Resistance training promotes cognitive and functional brain plasticity in seniors with probable mild cognitive impairment. Arch Intern Med 2012;172:666-8.

16 Law TD, Clark LA, Clark BC. Resistance exercise to prevent and manage sarcopenia and dynapenia. Annu Rev Gerontol Geriatr 2016;36:205-28.

$17 \mathrm{Yu}$ J. The etiology and exercise implications of sarcopenia in the elderly. Int J Nurs Stud 2015;2:199-203.

18 Cassilhas RC, Lee KS, Venâncio DP, et al. Resistance exercise improves hippocampus-dependent memory. Braz J Med Biol Res 2012;45:1215-20.

19 Kido K, Ato S, Yokokawa T, et al. Acute resistance exercise-induced IGF1 expression and subsequent GLUT4 translocation. Physiol Rep 2016;4:e12907.

20 Guan J, Mathai S, Liang H-, et al. Insulin-Like growth factor-1 and its derivatives: potential pharmaceutical application for treating neurological conditions. Recent Pat CNS Drug Discov 2013;8:142-60.

21 Friedrich N, Thuesen B, Jørgensen $\mathrm{T}$, et al. The association between IGF-1 and insulin resistance. Diabetes Care 2012;35:768-73.

22 Schneider HJ, Friedrich N, Klotsche J, et al. Prediction of incident diabetes mellitus by baseline IGF1 levels. Eur J Endocrinol 2011;164:223-9.

23 Bell ML, Whitehead AL, Julious SA. Guidance for using pilot studies to inform the design of intervention trials with continuous outcomes. $J$ Clin Epidemiol 2018;10:153-7.

24 Washburn RA, Smith KW, Jette AM, et al. The physical activity scale for the elderly (PASE): development and evaluation. J Clin Epidemiol 1993;46:153-62.

25 Guralnik JM, Simonsick EM, Ferrucci L, et al. A short physical performance battery assessing lower extremity function: association with self-reported disability and prediction of mortality and nursing home admission. J Gerontol 1994;49:85-94.

26 Podsiadlo D, Richardson S. The timed "Up \& Go": a test of basic functional mobility for frail elderly persons. J Am Geriatr Soc 1991;39:142-8.

27 Groll D, To T, Bombardier C, et al. The development of a comorbidity index with physical function as the outcome. J Clin Epidemiol 2005;58:595-602.

28 Yesavage JA, Brink TL, Rose TL, et al. Development and validation of a geriatric depression screening scale: a preliminary report. $J$ Psychiat Res 1982;17:37-49.

29 Balke B. A simple field test for the assessment of physical fitness. Rep Civ Aeromed Res Inst US 1963:1-8.

30 Folstein MF, Folstein SE, McHugh PR. "Mini-mental state". A practical method for grading the cognitive state of patients for the clinician. J Psychiatr Res 1975;12:189-98.

31 Nasreddine ZS, Phillips NA, Bédirian V, et al. The Montreal cognitive assessment, MoCA: a brief screening tool for mild cognitive impairment. J Am Geriatr Soc 2005;53:695-9.

32 Callisaya ML, Daly RM, Sharman JE, et al. Feasibility of a multimodal exercise program on cognition in older adults with type 2 diabetes - a pilot randomised controlled trial. BMC Geriatr $2017 ; 17$.

33 Falck RS, Davis JC, Milosevic E, et al. How much will older adults exercise? A feasibility study of aerobic training combined with resistance training. Pilot Feasibility Stud 2017;3.

34 Rosen WG, Mohs RC, Davis KL. A new rating scale for Alzheimer's disease. Am J Psychiatry 1984;141:1356-64.

35 Sánchez-Cubillo I, Periáñez JA, Adrover-Roig D, et al. Construct validity of the TRAIL making test: role of task-switching, working memory, inhibition/interference control, and visuomotor abilities. J Int Neuropsychol Soc 2009;15:438-50.

36 Guise BJ, Thompson MD, Greve KW, et al. Assessment of performance validity in the Stroop color and word test in mild traumatic brain injury patients: a criterion-groups validation design. $J$ Neuropsychol 2014;8:20-33.

37 Lezak MD. Neuropsychological assessment. 2nd ed. New York: Oxford University Press, 1983.

38 Wechsler D. Wechsler adult intelligence scale - revised. San Antonio, TX: Psychological Corporation, 1981.

39 Bastin C, Bahri MA, Miévis F, et al. Associative memory and its cerebral correlates in Alzheimer's disease: evidence for distinct deficits of relational and conjunctive memory. Neuropsychologia 2014;63:99-106.

40 Colcombe SJ, Kramer AF, Erickson KI, et al. Cardiovascular fitness, cortical plasticity, and aging. Proc Natl Acad Sci U S A 2004;101:3316-21. 